\title{
THE ADHERENCE OF SILICONE OIL TO STANDARD AND HEPARIN-COATED PMMA INTRAOCULAR LENSES
}

\author{
M. BATTERBURY ${ }^{1}$, D. WONG ${ }^{1}$, R. WILLIAMS ${ }^{2}$ and R. BATES \\ Liverpool
}

\begin{abstract}
SUMMARY
During combined cataract extraction and intraocular lens insertion in eyes undergoing removal of silicone oil, we noted that oil became adherent to the implant. This adhesion persisted post-operatively. Although patients seemed not to be symptomatic as a result of this effect, the oil interferes with the fundal examination. Since heparincoated intraocular lenses are more hydrophilic than unmodified lenses, oil should spread less well on these lenses and therefore be less adherent. This study was conducted to test this hypothesis. Heparin-surface-modified and unmodified polymethylmethacrylate (PMMA) lenses were immersed in 1000 centistoke neat silicone oil and in an emulsion of silicone oil taken from a patient. The lenses were washed with saline solution in an attempt to remove adherent oil. The lenses were photographed for examination and qualitative comparison. It was found that both neat and emulsified silicone oil was strongly adherent to both surface-modified and unmodified lenses, and could not easily be washed off. The heparin-coated lenses showed a tendency for adherence of emulsified oil. It is concluded that heparin coating of intraocular lenses does not prevent, though may reduce, oil adherence. We recommend that care be taken to avoid contact between oil and implant during combined oil removal and cataract extraction.
\end{abstract}

Cataract formation is a frequent complication associated with the use of silicone oil in retinal detachment surgery. In a retrospective review of 120 eyes, Franks and Leaver' showed that although cataract formation was delayed by early removal of silicone oil, after 2 years the majority of eyes had undergone surgery for cataract or had developed lens opacities. The surgeon is faced with several choices when dealing with cataract in silicone-oil-filled eyes: cataract extraction can be performed with or without intraocular lens (IOL) implantation; and the oil can be removed before, after or at the same time as the cataract extraction.

From: St Paul's Eye Unit, Royal Liverpool University Hospital, Liverpool; ${ }^{2}$ Department of Clinical Engineering, Duncan Building, Royal Liverpool University Hospital, Liverpool, UK.

Correspondence to: D. Wong, St Paul's Eye Unit, Royal Liverpool University Hospital, Liverpool L7 8XP, UK.
When extracapsular cataract extraction and IOL implantation were combined with oil removal, we observed that the oil often came into contact with the IOL. Droplets of oil adhered to the IOL and remained adherent throughout post-operative follow-up (Figs. 1,2). We used three-piece IOLs made of polymethylmethacrylate (PMMA). PMMA is a relatively hydrophobic material and, since silicone oil is a liquid with a low surface tension, it will spread and attach strongly to the implant surface. Surface-modified lenses are available which have a heparin coating. It has been shown that inflammatory cells are less likely to adhere to these surface-modified IOLs. ${ }^{2}$ The bound heparin renders the PMMA surface more hydrophilic, ${ }^{3}$ which will influence the interaction between the material surface and the silicone oil, such that the oil should spread less and therefore be less strongly adherent to the IOL.

This study was designed to investigate the influence of bound heparin on the interaction between silicone oil and PMMA IOLs.

\section{MATERIALS AND METHODS}

One thousand centistoke silicone oil supplied by Moor-

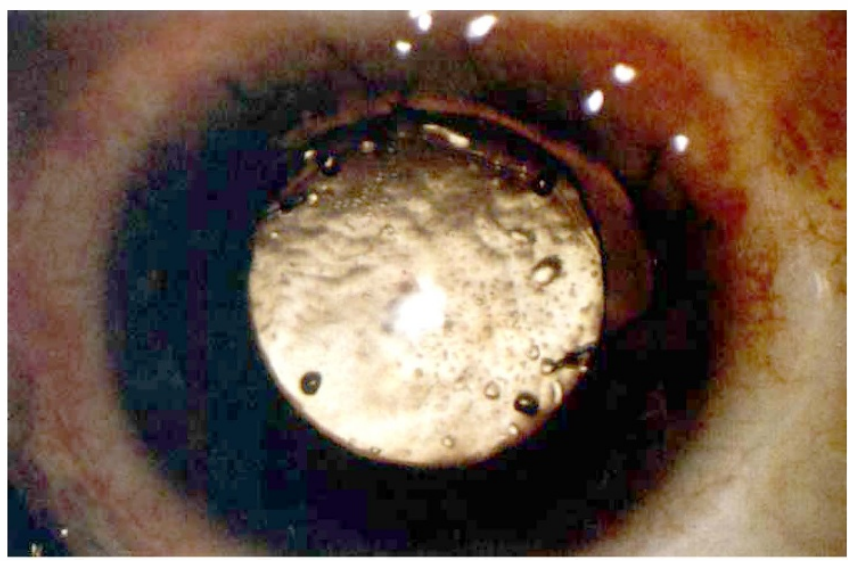

Fig. 1. Intraocular lens (IOL) coated with silicone oil immediately following insertion at combined oil removal and extracapsular cataract extraction (ECCE) with IOL implantation. 


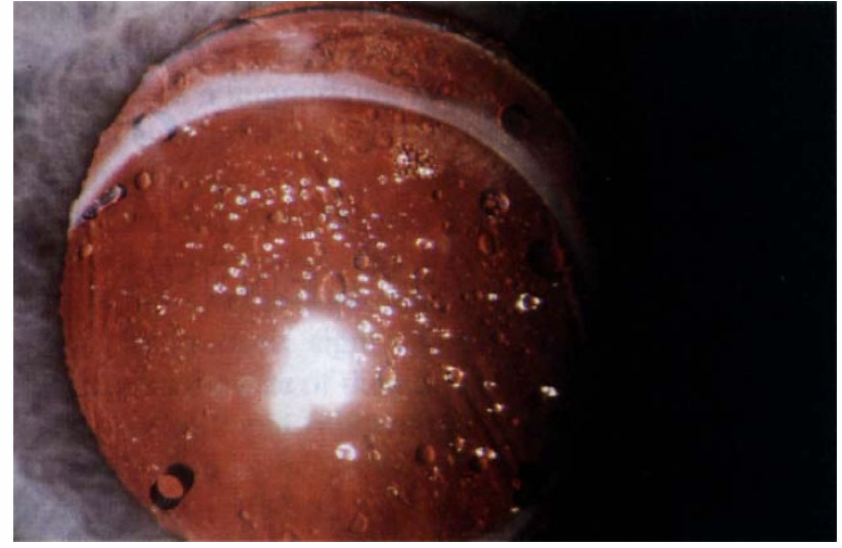

Fig. 2. Droplets of emulsified oil on an IOL.

fields Eye Hospital was used. Three one-piece PMMA IOLs without surface modification (Pharmacia) and three heparin-surface-modified PMMA IOLs (Pharmacia) were tested. Two techniques for studying oil-IOL interaction were used: (1) immersion of IOLs in neat silicone oil, (2) immersion of IOLs in a suspension of emulsified silicone oil droplets in saline solution. The emulsified droplets were taken from a patient who had undergone oil removal for emulsification.

The IOLs were incubated for 24 hours and then photographed. The lenses were then washed vigorously with $5 \mathrm{ml}$ balanced salt solution (BSS, Alcon) using a lacrimal cannula attached to a syringe. The lenses were photographed again. A qualitative assessment was made of the nature and degree of the oil-IOL interaction.

\section{RESULTS}

Following IOL immersion in neat oil the silicone oil spread on both the unmodified and the heparin-coated lenses. The oil could not be washed off (Fig. 3). After IOL immersion in a suspension of emulsified droplets, emulsified droplets adhered to both the unmodified PMMA and the heparin-coated PMMA lenses (Fig. 4). The number of droplets was noticeably less with the surface-modified lenses. Washing failed to remove the droplets.

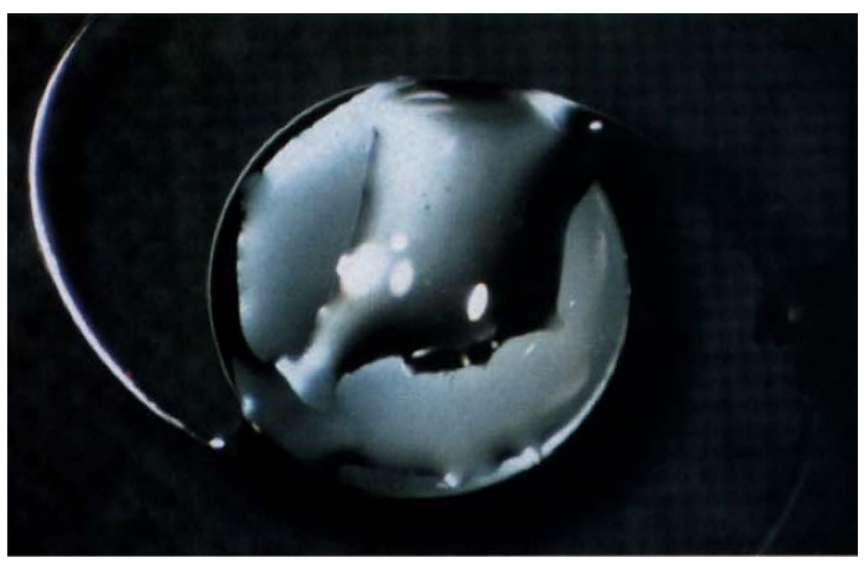

Fig. 3. Heparin-coated polymethylmethacrylate (PMMA) IOL after incubation with neat silicone oil. Irrigation failed to remove the oil from the IOL surface.

\section{DISCUSSION}

Our experiments confirmed our clinical observation that once silicone oil becomes attached to PMMA intraocular lenses, the adherence is strong and may be permanent. Clinically the droplets of silicone oil may degrade the optical property of the IOL, and impair fundus examination. Whilst patients complain of the visual effects of emulsified silicone oil droplets, they do not seem to notice the presence of oil droplets on the implant surface. This is likely to be because droplets circulating in the vitreous cavity cause changing scatter of incident light, which is more noticeable than light scatter due to static droplets.

When oil removal is combined with IOL implantation, it is recommended that the fornices be cleared of silicone oil by irrigation before IOL implantation. The aim should be to avoid contact of the IOL with oil.

There are clinical factors to suggest that oil removal and cataract extraction should be performed as separate procedures. Oil removal is associated with a significant risk of retinal re-detachment. ${ }^{1 . t-6}$ Further surgery for redetachment may be more difficult if the integrity of the globe is weakened by a recent cataract incision. Combined oil removal with lens implantation also leads to added complications such as formation of posterior synechiae, pupil capture of the IOL optic, accelerated posterior capsule opacification and pupil block glaucoma. These complications may be more common in patients with diabetes mellitus. ${ }^{7}$

Kampik et al. ${ }^{6}$ reported performing combined oil removal with extracapsular cataract extraction and IOL implantation (ECCE/IOL) in 30 eyes but did not comment on the complications; nevertheless, combined surgery was recommended. Removing silicone oil and performing cataract extraction as separate procedures will avoid contact of the IOL with the oil. It will also allow the surgeon to evaluate the visual potential, and manage the aphakia accordingly.

Moisseiev et al. ${ }^{.}$described a technique of cataract extraction without loss of silicone oil in which silicone oil removal is not indicated, and advised against IOL implantation. ECCE by aspiration in the presence of an anterior

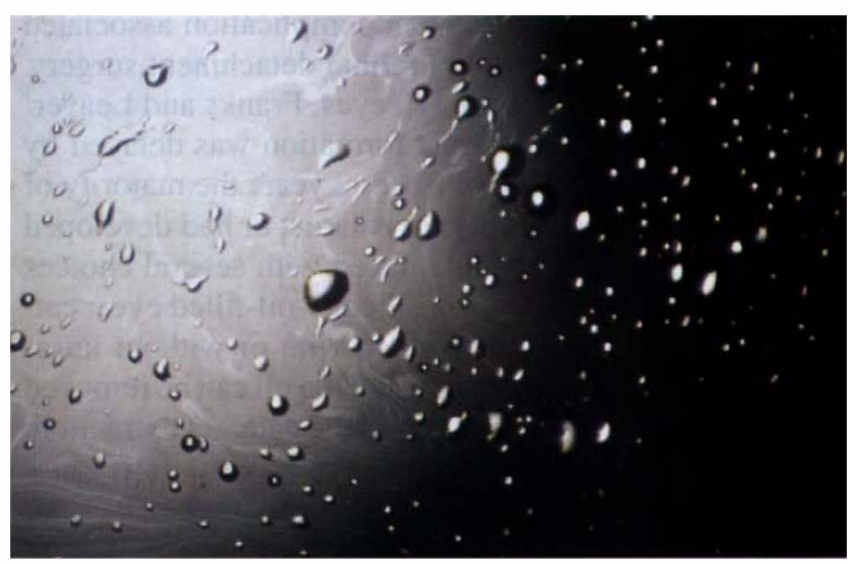

Fig. 4. Heparin-coated IOL after incubation in a suspension of emulsified silicone oil droplets in saline solution. Irrigation failed to remove the oil droplets from the IOL surface. 
chamber maintainer is followed by removal of residual capsule. No reference was made to procedures of combined oil removal and ECCE/IOL. These authors advised against standard extracapsular surgery due to the high incidence of accelerated posterior capsule opacification which impairs fundal examination, even after central laser capsulotomy. However, removal of the entire capsule makes secondary posterior chamber lens implantation more difficult in the eye with useful central vision.

Once emulsification has occurred in an oil-filled eye, it is difficult to remove all the droplets. A triple fluid-air exchange has been recommended to remove the droplets as completely as possible (J. L. Federman, personal communication). Despite this, there are often emulsified droplets left in an eye after oil removal. These droplets appear to be physically stable and can be seen in the anterior chamber as a fine emulsion simulating inflammatory cells. They come into contact with and adhere to the IOL. It may be more appropriate to use 5000 centistoke silicone oil, which is less likely to emulsify," in eyes in which subsequent oil removal and lens implantation are envisaged.

In the laboratory we demonstrated that silicone oil coated both the PMMA and the heparin-coated PMMA IOLs, even though the surface-modified IOLs have a more hydrophilic surface. Following incubation with emulsified oil droplets, the oil was seen to adhere to both types of lens. Although fewer droplets were observed on the heparin-coated IOL, the oil could not be washed off either IOL, suggesting that there was a strong attachment between the IOL and the silicone oil. Ejection of $5 \mathrm{ml}$ BSS through a lacrimal cannula would be expected to displace particles from the IOL surface.

Our work suggests that from a thermodynamic point of view the interfacial energy of the oil-PMMA interface is lower than that of the oil-aqueous interface, even when the PMMA has been modified by the bound heparin. In other words, there is a driving force for the oil to attach to the PMMA. This driving force is less strong for the heparin-bound surface, which could explain why fewer droplets were observed to be adherent to these lenses.

We thank Pharmacia for providing us with the lenses used in these experiments.

None of the authors has a proprietary interest in the development or marketing of the intraocular lenses described in this report.

Key words: Cataract surgery. Intraocular lens implantation, Silicone oil, Vitreoretinal surgery.

\section{REFERENCES}

1. Franks WA, Leaver PK. Removal of silicone oil: rewards and penalties. Eye 1991;5:333-7.

2. Borgioli M, Coster DJ, Fan RFT, Henderson J, Jacobi KW, Kirkby GR, Lai Y-K, Menezo JL, Montard M, Strobel J, Wollensak J. Effect of heparin surface modification of polymethylmethacrylate intraocular lenses on signs of postoperative inflammation after extracapsular cataract extraction. Ophthalmology 1992;99:1248-55.

3. King RN, Andrade D, Ma SM, Gregonis DE, Brostrom LR. Interfacial tensions at acrylic hydrogel-water interfaces. J Colloid Interface Sci 1985;103:62-75.

4. Casswell AG, Gregor ZJ. Silicone oil removal. II. Operative and postoperative complications. $\mathrm{Br} \quad \mathrm{J}$ Ophthalmol 1987;71:898-902.

5. Federman JL, Schubert HD. Complications associated with the use of silicone oil in 150 eyes after retina-vitreous surgery. Ophthalmology 1988;95:870-6.

6. Kampik A, Hoing C, Heidenkummer H-P. Problems and timing in the removal of silicone oil. Retina 1992; 12(Suppl):S11-6.

7. Pearson RV, McLeod D, Gregor ZJ. Removal of silicone oil following diabetic vitrectomy. $\mathrm{Br} \mathrm{J}$ Ophthalmol 1993;77:204-7.

8. Moisseiev J, Bartov E, Cahane M, Blumenthal M, Treister G. Cataract extraction in eyes filled with silicone oil. Arch Ophthalmol 1992;110:1649-51.

9. Heidenkummer H-P, Kampik A, Thierfelder S. Experimental evaluation of in vitro stability of purified polydimethylsiloxanes (silicone oil) in viscosity ranges from 1000 to 5000 centistokes. Retina 1992;12(Suppl):S28-32. 